\title{
Retraction of Characterization of Hydroxymethylation Patterns in the Promoter of $\beta$-globin Clusters in Murine Fetal Livers (DOI: 10.1089/dna.2014.2773)
}

\begin{abstract}
The online-ahead-of-print published article, Characterization of Hydroxymethylation Patterns in the Promoter of $\beta$-globin Clusters in Murine Fetal Livers (DOI: 10.1089/dna.2014.2773), is being officially retracted from DNA and Cell Biology due to scientific misconduct.

The senior author of the article, Yihang Shen, made use of data that he previously used as partial fulfillment of his PhD degree at Shanghai Jiao Tong University School of Medicine (Shanghai, People's Republic of China) where the work had been conducted and funded, and submitted the paper without permission from his laboratory director, Professor Fanyi Zeng, and without acknowledgment of all those involved in the study. Furthermore, the three co-first-authors listed in the published article did not participate in the study, and the scientists who should have been listed were omitted. Lastly, the funding source listed in the article was irrelevant, whereas the real funding sources were not disclosed or reported.
\end{abstract}

DNA and Cell Biology is dedicated to upholding the strictest standards of scientific publishing, and will not tolerate any improprieties. 